\title{
Amphiregulin Promotes Vascular Endothelial Growth Factor-C Expression and Lymphangiogenesis through STAT3 Activation in Human Chondrosarcoma Cells
}

\author{
Yu-Wen Huang ${ }^{a}$ Hsiao-Chi Tsai ${ }^{a}$ Shih-Wei Wang ${ }^{b}$ Shu-Jui Kuoc,d \\ Yi-Chin Fong ${ }^{\mathrm{e}, \mathrm{f}} \quad$ Chih-Hsin Tang ${ }^{\mathrm{a}, \mathrm{c}, \mathrm{g}, \mathrm{h}}$
}

\begin{abstract}
${ }^{a}$ Graduate Institute of Biomedical Sciences, China Medical University, Taichung, Taiwan, ${ }^{b}$ Department of Medicine, Mackay Medical College, New Taipei City, Taiwan, 'School of Medicine, China Medical University, Taichung, Taiwan, dDepartment of Orthopedic Surgery, China Medical University Hospital, Taichung, Taiwan, eDepartment of Sports Medicine, College of Health Care, China Medical University, Taichung, Taiwan, 'Department of Orthopaedic Surgery, China Medical University Beigang Hospital, Beigang, Taiwan, 9Department of Biotechnology, College of Health Science, Asia University, Taichung, Taiwan, ${ }^{h}$ Chinese Medicine Research Center, China Medical University, Taichung, Taiwan
\end{abstract}

\section{Key Words}

Amphiregulin • Chondrosarcoma $\cdot$ VEGF-C $・$ Lymphangiogenesis

\begin{abstract}
Background/Aims: Chondrosarcoma is the second most common primary malignancy of bone, characterized by a high metastatic potential. Increasing clinical data highlight the important role played by lymphangiogenesis in cancer metastasis. Amphiregulin (AR) has been implicated in tumor metastasis and lymphangiogenesis, but its association with vascular endothelial growth factor-C (VEGF-C) expression and lymphangiogenesis in chondrosarcoma is unclear. Methods: We used qPCR, ELISA and Western blotting to detect AR-induced VEGF-C expression in chondrosarcoma cells. Lymphangiogenesis was investigated by lymphatic endothelial cells (LECS) migration and tube formation. An in vivo experiment examined AR expression in tumor-associated lymphangiogenesis. Results: In this study, we found that both AR and VEGF-C expression correlated with tumor stage and were significantly higher than levels found in normal cartilage. Exogenous AR promoted VEGF-C expression in chondrosarcoma cells in a time- and dose-dependent manner and subsequently increased migration and tube formation of LECs. AR also increased VEGF-C expression and lymphangiogenesis through the SrC/MEK/ERK/STAT3 signaling pathway. However, it is unclear as to how an EGFR ligand $(A R)$ induces activation of the Src kinase. Knockdown of AR decreased VEGF-C expression in chondrosarcoma cells. Similarly, lymphangiogenesis was abolished in AR knockdown cells
\end{abstract}


in an in vivo model of chondrosarcoma. Conclusion: These results indicate that AR occurs through the SrC/MEK/ERK/STAT-3 pathway, activating VEGF-C expression and contributing to lymphangiogenesis in human chondrosarcoma. Thus, AR could be a therapeutic target in metastasis and lymphangiogenesis of chondrosarcoma.

\section{Introduction}

Chondrosarcoma is a malignant cartilaginous tumor of bone and soft tissue. The most common sites of involvement are in cartilage-abundant bone, such as the femur, tibia, or pelvis. Chondrosarcoma typically affects adults aged 20-60 years and is more common in men. Patients may be able to feel an abnormal bony bump, which can vary in size and location; most individuals will experience pain, swelling, or limited movement. Treatment involves complete surgical removal, radiation therapy [1, 2] and chemotherapy [3]. We have previously reported that chondrosarcomas can easily metastasize to the lung, liver [4] and the other organs. Distant metastasis is associated with highly malignant potential and a poor prognosis [3]. Therefore, novel therapeutic strategies are needed to treat human chondrosarcoma metastasis.

Tumor-induced lymphangiogenesis is crucial for promoting the ability of cancer cells to migrate, invade, proliferate and develop distant metastasis [5-8]. Vascular endothelial growth factor-C (VEGF-C) is a well-characterized lymphangiogenic factor involved in lymph formation, tumor growth, lymphangiogenesis and metastasis [7, 9] in various types of human cancers, such as breast cancer [10], colorectal [11, 12] and ovarian cancer [9], chondrosarcomas [13] and lung cancer [14]. Moreover, VEGF-C promotes the growth and survival of lymphatic endothelial cells (LECs).

Amphiregulin (AR) is a ligand of the epidermal growth factor receptor (EGFR). Initially synthesized as a transmembrane precursor, AR is then proteolytically processed to its mature secreted form [15]. AR-EGFR signaling mediates the migration of bone marrow mesenchymal progenitors to parathyroid hormone (PTH) $[16,17]$. AR plays an important role in tumor pathogenesis and metastasis, including colorectal cancer, osteosarcoma, and breast cancer [18-20]. Evidence also suggests that AR may serve as a novel resistance factor in cancer cells $[18,21,22]$. We have previously reported that AR promotes the expression of $\alpha 6 \beta 1$ integrin and cell motility of human chondrosarcoma cells [4]. In another paper, we have reported that AR enhances VEGF-A expression and promotes the angiogenesis of human endothelial progenitor cells [23], which implies that AR is involved in the metastatic process of chondrosarcoma.

It is known that $\mathrm{AR}$ increases the activity of E-cadherin transcription protein expression through the ERK pathway, promoting tumor invasion and proliferation in ovarian cancer [24]. In breast cancer, it has been established that VEGF-C enhances tube formation and CXCL12 promotes lymphangiogenesis of LECs via the NF- $\mathrm{BB}$ signaling pathway [7]. Up until now, no such association has been observed between AR and VEGF-C expression in human chondrosarcoma. In this study, we found that AR enhances VEGF-C production and promotes lymphangiogenesis through the Src/MEK/ERK/STAT3 signaling pathway.

\section{Materials and Methods}

\section{Materials}

Recombinant human AR was purchased from R\&D Systems (Minneapolis, MN, USA) and Trizol was obtained from Life Technologies. DMEM, $\alpha$-MEM, fetal bovine serum (FBS), and remaining cell culture reagents were bought from Gibco-BRL life technologies. p-Src- and p-MEK-specific rabbit polyclonal antibodies were bought from Cell Signaling Technology. Santa Cruz Biotechnology supplied rabbit polyclonal antibodies specific for ERK and p-STAT3. Mouse polyclonal antibodies specific for STAT3, p-ERK, Src, MEK, $\beta$-actin and VEGF-C were bought from Santa Cruz Biotechnology. LYEV-1 antibody was obtained from 


\section{Cellular Physiology Cell Physiol Biochem 2019;52:1-15 \\ \begin{tabular}{ll|l}
\cline { 2 - 2 } DOl: 10.33594/000000001 & (c) 2019 The Author(s). Published by
\end{tabular} \\ \begin{tabular}{l|l} 
Published online: 18 February 2019 Cell Physiol Biochem Press GmbH\&Co. KG \\
\hline
\end{tabular} \\ Huang et al:: Amphiregulin Induces VEGF-C-Dependent Lymphangiogenesis}

Abcam. Src, MEK and ERK small interfering RNAs (siRNAs) were obtained from Dharmacon Research. The AR-shRNA plasmid CCGGGAACGAAAGAAACTTCGACAACTCGAGTTGTCGAAGTTTCTTTCGTTCTTTTTG was purchased from RNAiCore (Taipei, Taiwan).

\section{Cell culture}

Human chondrosarcoma cell lines JJ012 and SW1353 were supplied by Dr. Sean P. Scully's laboratory (University of Miami School of Medicine, Miami, FL) and The American Type Culture Collection (Manassas, VA, USA), respectively. The cells were cultured in Dulbecco's modified Eagle's medium (DMEM) $/ \alpha$-MEM supplemented with $10 \% \mathrm{FBS}$ and $100 \mathrm{U} / \mathrm{mL}$ penicillin/streptomycin in a chamber atmosphere of $5 \% \mathrm{CO}_{2}$ at $37^{\circ} \mathrm{C}$. The human telomerase-immortalized human dermal lymphatic endothelial cell line (hTERT-HDLEC) was purchased from Lonza (Walkersville, MD, USA). These cells stably express endothelial cell-specific markers, including CD31, and recognized lymphatic markers, including podoplanin. In addition, they can take up acetylated LDL and induce tube formation. We used the EGM-2MV BulletKit Medium (i.e., EBM-2 basal medium plus a SingleQuots kit) to grow human LECs. We seeded cells onto $1 \%$ gelatin-coated plastic ware and cultured them in a chamber atmosphere of $5 \% \mathrm{CO}_{2}$ at $37^{\circ} \mathrm{C}$.

\section{Preparation of conditioned medium and ELISA assay}

We pretreated cells with inhibitors for 30 min or transfected them with siRNAs for $24 \mathrm{~h}$, then stimulated the cells with AR for $24 \mathrm{~h}$. Conditioned medium (CM) was collected post-treatment and stored at $-80^{\circ} \mathrm{C}$ until use. We determined VEGF-C levels with the VEGF-C enzyme immunoassay kit, according to the manufacturer's instructions.

\section{Transwell migration assay}

LECs were seeded in the upper chambers of Transwell inserts ( $8 \mu \mathrm{m}$ pore size; Costar, NY, USA) in 24well plates $\left(7 \times 10^{4}\right.$ per well). CM $(300 \mu \mathrm{L})$ was placed in the lower chambers. After $16 \mathrm{~h}$ of incubation, we wiped off the cells from the top of the membrane using cotton swabs. Migrant cells were collected from the bottom of the membrane then fixed with $37 \%$ formaldehyde and stained with crystal violet before being counted by microscopy.

\section{Tube formation assay}

Matrigel was dissolved overnight at $4^{\circ} \mathrm{C}$, cultured in 48 -well plates (150 $\mu \mathrm{L}$ per well), then incubated for $30 \mathrm{~min}$ at $37^{\circ} \mathrm{C}$. We resuspended LECs at a density of $3 \times 10^{5} / 100 \mu \mathrm{L}$ in culture medium $(50 \%$ EGM-2MV BulletKit Medium and 50\% chondrosarcoma cell $\mathrm{CM}$ ) and mixed with different treatment CMs before adding them to the wells. The cells were incubated for $6 \mathrm{~h}$ at $37^{\circ} \mathrm{C}$. We assessed LEC tube formation by microscopy and photographed each well. We calculated tube branches and tube lengths with MacBiophotonics Image J software.

\section{Western blot analysis}

Chondrosarcoma cells were collected and lysed in cold RIPA buffer with protein inhibitors. Cell lysates were centrifuged and supernatant protein was quantified using a BCA protein assay kit. Proteins were resolved using SDS-PAGE and transferred to Immobilon polyvinyldifluoride (PVDF) membranes [25]. After blocking the blots with 4\% BSA for $1 \mathrm{~h}$ at room temperature, we probed them with rabbit/mouse anti-human antibodies against p-Src, p-MEK, p-STAT3 and p-ERK (1:1000) for a further hour at room temperature. Blots were washed three times then incubated with an anti-rabbit/mouse peroxidase-conjugated secondary antibody (1:1000) at room temperature for $1 \mathrm{~h}$ and visualized by Imagequant LAS 4000.

\section{Quantitative real-time PCR}

Total RNA from chondrosarcoma cells was extracted using Trizol reagent (Carlsbad, CA, USA) and reverse-transcribed into cDNA using M-MLV Reverse Transcriptase, Oligo (dT), and dNTP Mix (Promega, Madison, WI), as per the manufacturer's instructions. Applied Biosystems supplied sequences for all target gene primers and probes. qPCR assays were repeated three times using the StepOnePlus sequence detection system (Applied Biosystems). Relative gene expression levels were calculated using glyceraldehyde-3phosphate dehydrogenase (GAPDH) expression patterns. 


\section{Chromatin immunoprecipitation (ChIP)}

ChIP was performed as previously described [26]. DNA was immunoprecipitated using an anti-STAT3 antibody, extracted, purified and resuspended in $\mathrm{H}_{2} \mathrm{O}$. We used immunoprecipitated DNA as a template for PCR, using the following primer specific for the 162-bp VEGF-C promoter fragment between 8 and 169 (contains putative STAT3 binding sites): 5'-CACAGACCAAGGGAGAGAGG-3' and 5'-CACTCCCGAGTTCTTGTCCT-3'. We used $1 \%$ agarose gel to resolve PCR products by electrophoresis and visualized the segments with ultraviolet light.

In vivo tumor xenograft study and immunohistochemistry analysis

Male SCID mice (4 weeks old; BALB/c-nu [nu/nu]) were bought from Taipei's National Science Council Animal Center and maintained in pathogen-free conditions. JJ012 cells and JJ012/shAR cells were injected subcutaneously into both flanks of each animal, and tumors were allowed to grow for 1 month. To investigate the lymphangiogenesis effect in tumor tissues in vivo, paraffin-embedded tumor sections and human chondrosarcoma tissue arrays were prepared, mounted on slides, deparaffinized in xylene, rehydrated, and washed in distilled water. We incubated human tissue sections overnight at $4^{\circ} \mathrm{C}$ with VEGF-C or LYVE1 (1:100) primary antibodies, before incubating them with secondary antibody (1:100) for $1 \mathrm{~h}$ at room temperature. The sections were then stained with diaminobenzidine.

\section{Patients and specimen preparation}

China Medical University Hospital's Institutional Review Board approved the study protocol. Prior to enrollment, all patients completed written informed consent. We obtained tumor tissue specimens from patients undergoing surgical resection for chondrosarcoma at China Medical University Hospital.

\section{Statistical analysis}

The data are expressed as the mean \pm standard error of the mean (S.E.M.) from at least three independent experiments. Analysis used the two-tailed Student's $t$-test. A statistical probability of $P<0.05$ was considered significant. Statistical analysis was performed using GraphPad Prism 6.0.

\section{Results}

AR involves lymphangiogenesis in chondrosarcoma cells

We have previously shown that AR promotes tumor metastasis in chondrosarcoma cells [4]. In this study, we investigated whether AR influences VEGF-C expression and lymphangiogenesis in chondrosarcoma cells. We directly applied AR to human chondrosarcoma cell lines (JJ012 and SW1353) and examined VEGF-C expression. AR enhanced VEGF-C mRNA expression and VEGF-C production in a concentration-dependent and time-dependent manner (Fig. 1A-D). During tumor-induced lymphangiogenesis, lymphatic endothelial cells must undergo migration, proliferation and tube formation to form new lymph vessels [27]. We therefore examined whether AR-dependent VEGF-C expression stimulated LECs during lymphangiogenesis. Using VEGF-C as a positive control, we found that CM from AR-treated chondrosarcoma cells promoted tube formation and migration in LECs. Anti-VEGF-C monoclonal antibody (mAb) abolished AR-mediated effects, which suggests that AR induces lymphangiogenesis in a concentration-dependent manner, depending on the VEGF-C inhibitor (Fig. 1E and F). These data indicate that AR-dependent VEGF-C expression promotes lymphangiogenesis in human chondrosarcoma cells.

\section{AR promotes VEGF-C expression and lymphangiogenesis in chondrosarcoma cells through} the Src/MEK/ERK pathway

The Src pathway is involved in lymphangiogenesis and VEGF-C expression [28]. Treatment with an Src-specific inhibitor (PP2) or transfection with Src siRNA diminished AR-enhanced VEGF-C mRNA expression and protein production (Fig. 2A and B). CM from chondrosarcoma cells pretreated with an Src specific inhibitor (PP2) and Src siRNA inhibited LEC tube formation and migration (Fig. 2C and D). To examine the mechanism underlying AR- 
Fig. 1. $A R$ promotes VEGF-C expression and subsequently increases lymphangiogenesis in human chondrosarcoma cells. Cells were incubated with AR (3-50 $\mu \mathrm{g} / \mathrm{ml})$ for 24 $\mathrm{h}$ and then $\operatorname{AR}(50 \mu \mathrm{g} / \mathrm{ml})$ for 6,12 and $24 \mathrm{~h}$; VEGF-C expression was examined by qPCR (A and B) and ELISA (C and D). (E and F) JJ012 cells were incubated with AR (3-50 $\mu \mathrm{g} / \mathrm{ml})$ for $24 \mathrm{~h}$. The medium was collected as conditioned medium (CM) and JJ012 cells were pretreated with IgG/VEGF-C antibody (1 $\mathrm{mg} / \mathrm{ml})$ for $30 \mathrm{~min}$, then incubated with AR for $24 \mathrm{~h}$ before being applied to LECs. LEC migration and capillary-like structure formation was examined by Transwell and tube formation assay ( $\mathrm{E}$ and F). Results are expressed as the mean \pm SEM. ${ }^{*} \mathrm{P}<0.05$ compared with controls; $\# \mathrm{P}<0.05$ as compared with the AR-treated group.

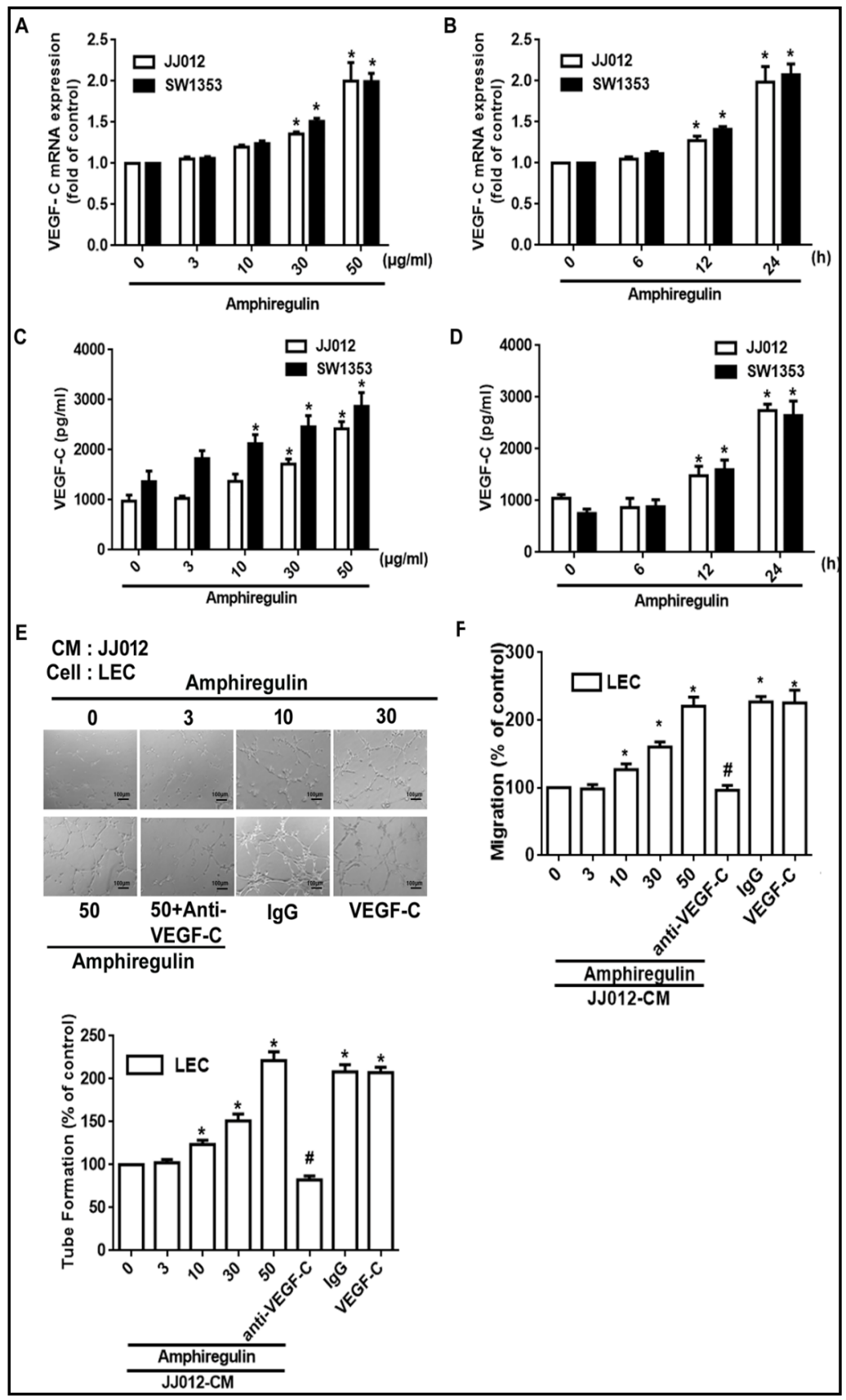

induced enhancement of VEGF-C expression, we measured Src protein phosphorylation after AR stimulation. In Western blot analysis, AR-stimulated chondrosarcoma cells increased Src phosphorylation in a time-dependent manner (Fig. 2E).

MEK/ERK is downstream of the c-Src signaling pathway [29, 30]. The MEK/ERK1/2 signaling pathway is involved in lymphatic metastatic spread in urinary bladder cancer [31]. We therefore investigated whether AR enhances VEGF-C expression via the MEK/ERK pathway. VEGF-C mRNA and protein expression were reduced after treatment with a MEK/ ERK inhibitor (PD98059/U0126) and transfection with MEK/ERK siRNA (Fig. 3A and B). CM containing the MEK/ERK inhibitor and siRNA inhibited LEC tube formation and migration (Fig. 3C and D). MEK and ERK phosphorylation were increased by AR in a time-dependent manner (Fig. 3E). Thus, AR increased VEGF-C expression and lymphangiogenesis in chondrosarcoma cells via the MEK/ERK pathway. Western blotting showed that AR-induced 
Fig. 2. The Src pathway is involved in AR-induced VEGF-C expression and lymphangiogenesis in human chondrosarcoma cells. Cells were pretreated with PP2 for 30 min or transfected with siRNAs for $24 \mathrm{~h}$, then stimulated with AR $(50 \mu \mathrm{g} / \mathrm{ml})$ for $24 \mathrm{~h}$. VEGF-C expression was examined by qPCR (A) and ELISA (B). The medium was collected as $\mathrm{CM}$ and then applied to the LECs. Capillary-like structure formation and LEC migration was examined by tube formation and the Transwell assay, respectively (C and D). (E) JJ012 cells were incubated with AR (50 $\mu \mathrm{g} / \mathrm{ml})$ for 120 min, and Src phosphorylation was determined by Western blotting. Western blot data were normalized to Src as the loading control. Results are expressed as the mean \pm SEM. ${ }^{*} \mathrm{P}<0.05$

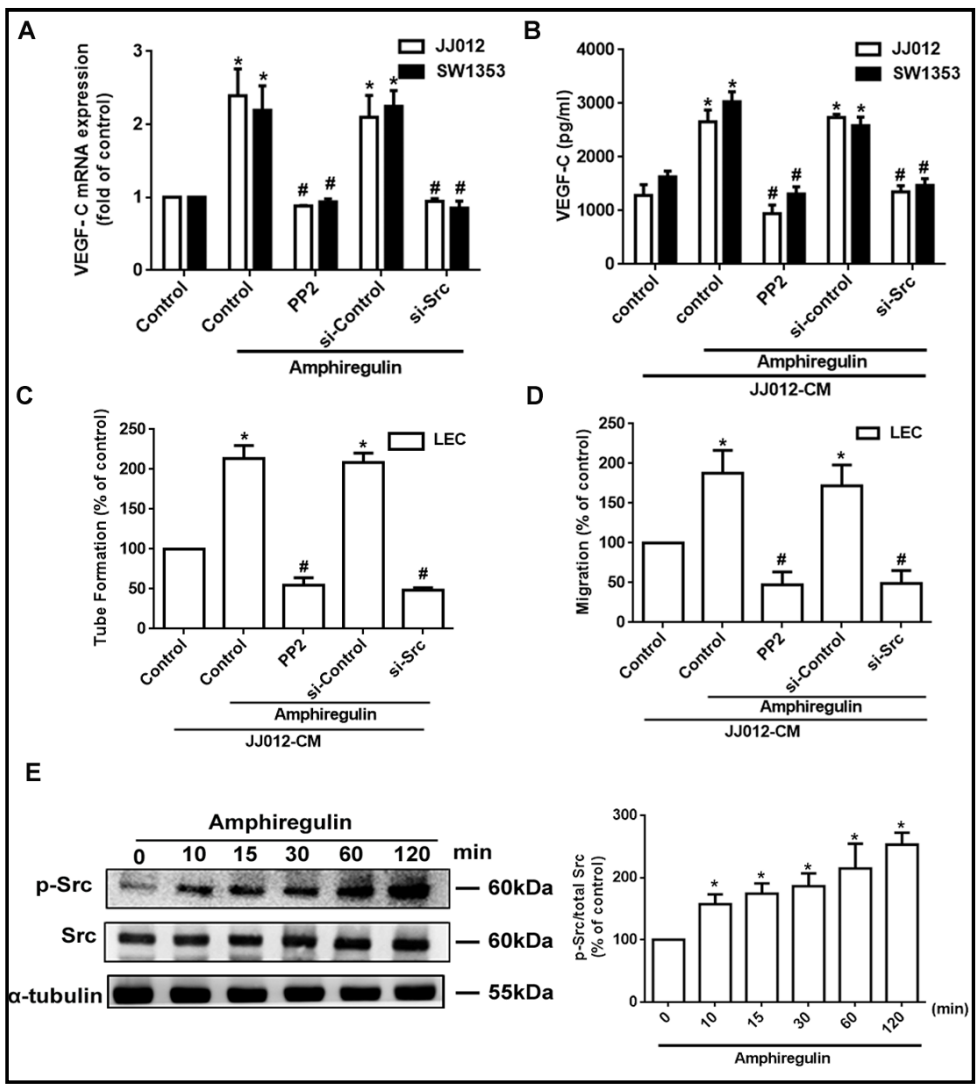
compared with controls; \#P<0.05 compared with the AR-treated group.

phosphorylation of MEK or ERK expression was inhibited by treatment with Src and MEK inhibitors, which suggests that c-Src is an upstream molecule in the MEK/ERK pathway (Fig. $3 F)$.

Transcription factor STAT3 is required for AR-promoted VEGF-C expression and lymphangiogenesis in human chondrosarcoma cells

STAT3 is a transcription factor that plays a pivotal role in the promotion of VEGF-C production in LECs [28] and tumor lymphangiogenesis [32]. Previous investigations have shown that STAT3 is downstream of the MEK/ERK signaling pathway [33]. We therefore analyzed the role of STAT3 in AR-induced increases in VEGF-C expression and lymphangiogenesis. We found that the STAT3 inhibitor and siRNA abolished STAT3 expression in chondrosarcoma cells. VEGF-C mRNA expression and protein production were significantly diminished after treatment with the STAT3 inhibitor and siRNA (Fig. 4A and B). The CM also reduced the result which AR-induce the tube formation and migration in LECs (Fig. 4C and D). STAT3 protein phosphorylation was increased after AR treatment (Fig. 4E). The data reveal that AR induces STAT3 activation in a time-dependent manner. Incubation with Src and MEK inhibitors antagonized AR-induced STAT3 phosphorylation (Fig. 4F), suggesting that STAT3 is downstream of the MEK/ERK pathway.

We further examined whether AR promotes STAT3 binding to the VEGF-C promoter region. The JASPAR database (http://jaspar.genereg.net/cgi-bin/jaspar_db.pl) implicates the STAT3 binding site in the VEGF-C sequence. We therefore constructed a primer that included the VEGF-C gene. Chromatin immunoprecipitation (ChIP) analysis showed that AR increased STAT3 recruitment to the 162-bp VEGF-C promoter fragment between 8 and 169 (contains putative STAT3 binding sites): 5'-CACAGACCAAGGGAGAGAGG-3' and 
Fig. 3. The MEK/ERK pathway is involved in AR-induced VEGF-C expression and lymphangiogenesis in human chondrosarcoma cells. Cells were pretreated with U0126 and PD98059 for 30 min or transfected with siRNAs for $24 \mathrm{~h}$, then stimulated with AR $(50 \mu \mathrm{g} / \mathrm{ml})$ for 24 h. VEGF-C expression was examined by qPCR (A) and ELISA (B). The medium was collected as CM and then applied to the LECs. Capillarylike structure formation and LEC migration was examined by tube formation and the Transwell assay, respectively (C and D). (E) JJ012 cells were incubated with AR (50 $\mu \mathrm{g} / \mathrm{ml}$ ) for 120 min, and MEK/ERK phosphorylation was determined by Western blotting. (F) JJ012 cells were pretreated with inhibitors as indicated, then incubated with AR for $60 \mathrm{~min}$ and analyzed by Western blotting with MEK and ERK antibodies. Western blot data were normalized to MEK and ERK as the loading control. Results are expressed as the mean \pm SEM. ${ }^{*} \mathrm{P}<0.05$ compared with control; \#P<0.05 compared with the AR-treated group.

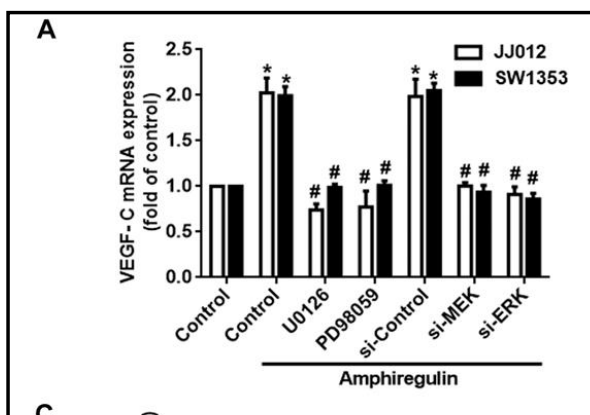

C $\overline{\bar{o}}_{300}$
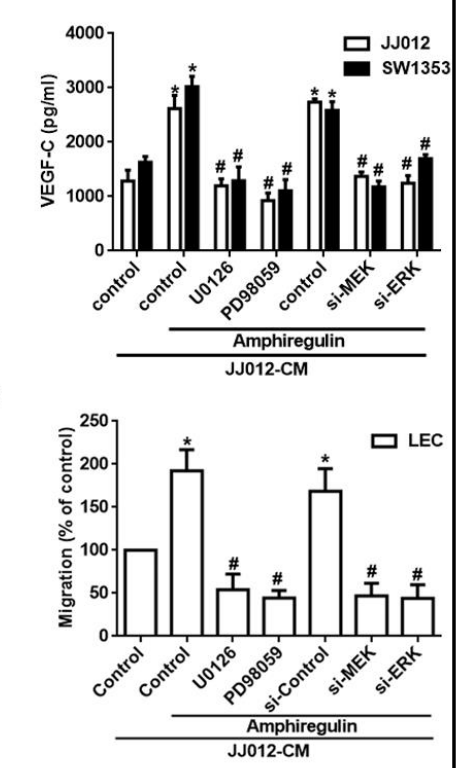

E

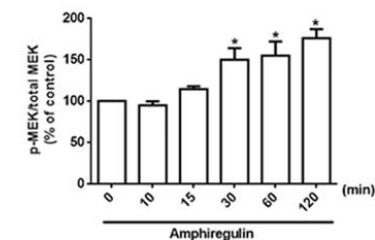

p-ERK $=-2-2 \mathrm{kDa}$

ERK $=0000=42 \mathrm{kDa}$

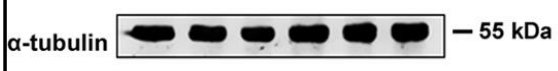

F
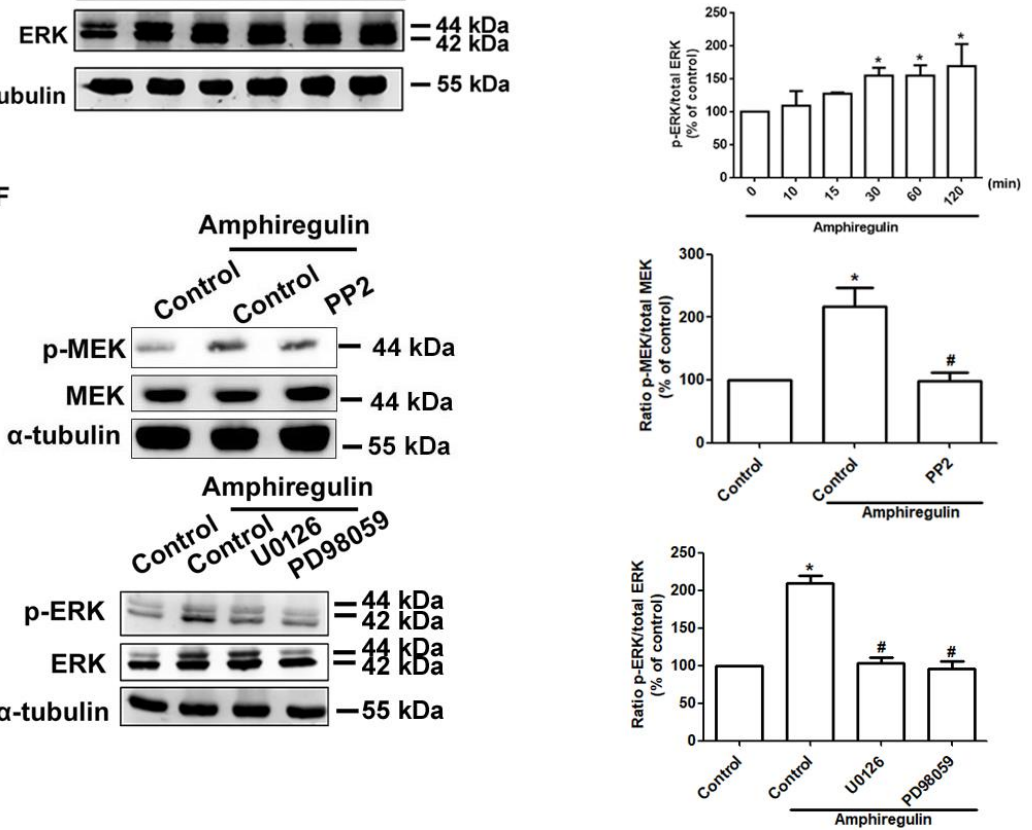

5'-CACTCCCGAGTTCTTGTCCT-3' (Fig. 4G). AR-induced STAT3 binding to the VEGF-C sequence was reversed by treatment with Src and MEK inhibitors PP2, U0126 and PD98059 (Fig. 4G). These observations suggest that AR enhances VEGF-C expression in chondrosarcoma cells through the Src-MEK/ERK/STAT3 signaling pathway. 
Fig. 4. $A R$ induced VEGF-C expression and lymphangiogenesis in human chondrosarcoma cells via STAT3 activation. Clls were pretreated with a STAT3 inhibitor for 30 min or transfected with siRNAs for $24 \mathrm{~h}$, then stimulated with AR $(50 \mu \mathrm{g} / \mathrm{ml})$ for 24 h. VEGF-C expression was examined by qPCR (A) and ELISA (B). The medium was collected as CM and then applied to the LECs. Capillary-like structure formation and LEC migration was examined by tube formation and Transwell assay, respectively (C and D). (E) JJ012 cells were incubated with AR $(50 \mu \mathrm{g} / \mathrm{ml})$ for $120 \mathrm{~min}$, and STAT3 phosphorylation was determined by Western blotting. (F) JJ012 cells were pretreated with inhibitors as indicated, then incubated with AR for $60 \mathrm{~min}$ and analyzed by Western blotting with STAT3 antibodies. Western blot data were normalized to STAT3 as the loading control. (G) Cells were pretreated with PP2, U0126 and PD98059 for 30 min, then stimulated with AR $(50 \mu \mathrm{g} / \mathrm{ml})$, and the chromatin immunoprecipitation assay was performed. Chromatin was immunoprecipitated with anti-STAT3 antibody. One percent of the precipitated chromatin was assayed to verify equal loading (input). Results are expressed as the mean $\pm \mathrm{SEM}$. ${ }^{*} \mathrm{P}<0.05$ compared with controls; $\# \mathrm{P}<0.05$ compared with the AR-treated group.

\section{Knockdown AR suppresses in vivo tumor lymphangiogenesis}

We have previously shown that stable JJ012/shAR cells silence AR in chondrosarcoma cells [4]. Here, we observed that production of VEGF-C mRNA and protein was inhibited in AR shRNA stable clones (Fig. 5A and B). Similarly, CM from JJ012/shAR-treated cells reduced LEC tube formation and migration (Fig. 5C and D). When we investigated the role of AR in tumor lymphangiogenesis, we found that knockdown AR reduced tumor VEGF-C and LYVE1 expression in paraffin-embedded tumor sections (Fig. 5E). Our findings show that AR enhances tumor-associated lymphangiogenesis in vivo. 
Fig. 5. Knockdown AR decreases VEGF-C expression and lymphangiogenesis in chondrosarcoma tumors. VEGF-C expression was detected in JJ012 and JJ012s-hAR cells by qPCR and ELISA (A and B). CM from JJ012 and JJ012shAR cells was applied to LECs and analyzed for tube formation activity (C) as well as migration activity (D). Mice were injected subcutaneously with Matrigel mixed with JJ012 and JJ012/ shAR cells, then were sacrificed after 28 days. Tumor sections were embedded in paraffin and $\mathrm{AR}$ and VEGF-C antibodies were used for immunostaining (E). The correlation between tumor stage, AR and VEGF-C (F). The correlation between $\mathrm{AR}$ and VEGF-C mRNA expression in chondrosarcoma tissue was examined by qPCR (G). Quantitative results are expressed as the mean \pm SEM. ${ }^{*} \mathrm{P}<0.05$ as compared with the control group.

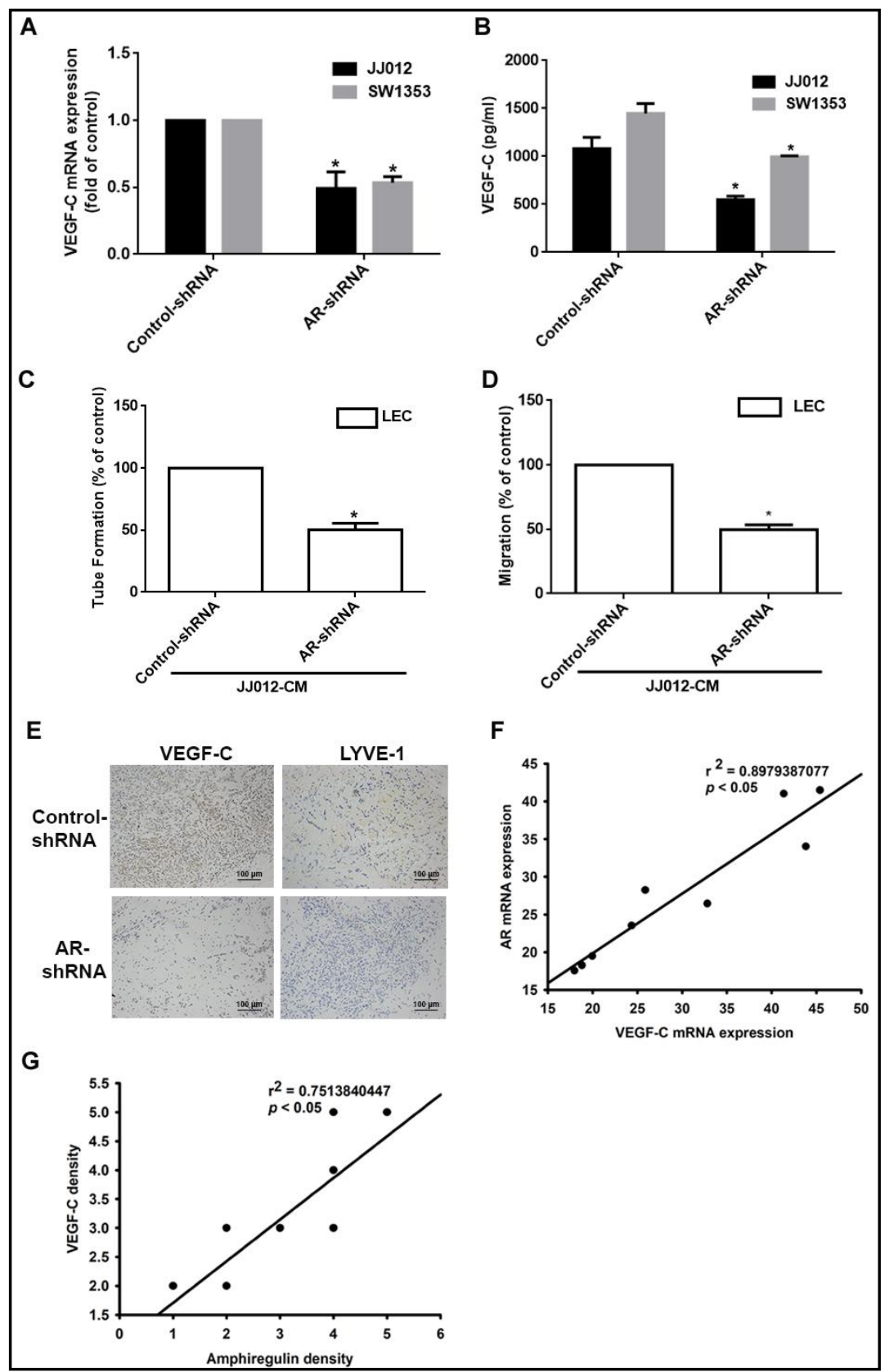

\section{Clinical correlation between $A R$ and VEGF-C}

To investigate AR expression in chondrosarcoma lymphangiogenesis, we analyzed AR and VEGF-C expression from human chondrosarcoma tissue. Our previous study demonstrated higher AR and VEGF-C mRNA expression in chondrosarcoma specimens than in normal cartilage and that the levels of expression correlated significantly with tumor stage $[13,23]$. We found a positive correlation between AR and VEGF-C mRNA expression (Fig. 5F). Quantitative data from the chondrosarcoma tissue array showed a highly positive relationship between AR and VEGF-C expression (Fig. 5G). These results reveal that AR is strongly associated with VEGF-C expression and tumor lymphangiogenesis in human chondrosarcoma cells. 


\section{Discussion}

The prognosis of chondrosarcomas depends on many variables, most importantly tumor grade and stage; high-grade tumors and axial/pelvic tumor location in high-grade chondrosarcoma are poor prognostic factors for overall survival, while the percentage of dedifferentiated component in dedifferentiated chondrosarcoma significantly influences disease-free survival [34, 35]. Lymphangiogenesis is an indispensable step for cancer metastasis, facilitating the generation of new lymphatic vessels that serve as conduits for tumor dissemination to lymph nodes [36-38]. Identifying target therapies is therefore essential for the management of lymphangiogenesis and metastasis associated with chondrosarcoma [39-41].

VEGF-C plays an essential role in lymphatic vessel formation and differentiation [8]. For instance, higher intratumoral VEGF-C expression is associated with significantly worse overall survival compared with low expression levels in ovarian cancer [9], while suppressing the NF- $\kappa B$ signaling pathway inhibits peritumoral lymphatic vessel development and reduces VEGF-C expression in triple-negative breast cancer cells [7].

The protein encoded by the $A R$ gene is a member of the EGF family. Upregulated $A R$ expression has been reported in many cancers, including colorectal [18], breast [42], lung [22], gastric [43, 44], and ovarian cancer [24]. Moreover, evidence suggests that AR plays an important role in tumor metastasis in chondrosarcoma [4] and resistance to therapy in HER2-positive breast cancer, reducing the antiproliferative effect of trastuzumab via AKT and ERK activation [22, 42]. Several studies have indicated that abnormal AR expression predicts poor outcome in patients [42, 44-46]. Non-small cell lung cancer (NSCLC) exosomes containing AR induce EGFR pathway activation, that in turn causes osteoclast differentiation and bone metastasis in lung cancer [47]. In gastric cancer, a positive correlation between Trop2 and AR increases tumor cell division and promotes metastasis, which suggests that Trop2 and AR could serve as prognostic biomarkers in gastric cancer [43]. In head and neck squamous cell carcinoma (HNSCC), increasing miR-34a expression attenuates AR levels and thus significantly decreases HNSCC invasion and metastasis via the ErbB signaling pathway [48]. We have previously revealed that AR promotes the ability of chondrosarcoma cells to migrate and metastasize [4]. In our present study, AR enhanced VEGF-C expression and promoted lymphangiogenesis of human chondrosarcoma cells. We found a positive correlation between AR and VEGF-C expression in tumor specimens from chondrosarcoma patients.

A number of studies have observed that c-Src, a cytoplasmic protein tyrosine kinase, is capable of enhancing cell proliferation, reducing apoptosis and promoting metastasis in chondrosarcoma $[49,50]$. Moreover, Src mediates ERK1/2 and p38MAPK activation, contributing to interleukin 6 (IL-6)-induced VEGF-Cexpression in murine LECs [50]. Inhibition of Src significantly reduces LEC migratory activity and suppresses lymphangiogenesis in vitro and in vivo [51]. Our current data show that AR enhances lymphangiogenesis through Src activation and VEGF-C expression in chondrosarcoma cells.

AR contains multiple domains, one of which is the heparin-binding (HB) domain, consisting of an EGF-like domain and a very basic $\mathrm{NH}_{2}$-terminal extension, which contains glycosylation sites and putative nuclear localization signals. AR binding to the extracellular domain of the EGF receptor (EGFR) influences cell proliferation and causes EGFR autophosphorylation of the EGFR tyrosine kinase, and a number of cellular substrates to undergo tyrosine phosphorylation $[15,52]$. When EGFR undergoes phosphorylation at the plasma membrane, the receptor activation induces phosphorylation of key tyrosine residues within the $\mathrm{COOH}$-terminal portion of EGFR and provides specific docking sites for cytoplasmic proteins containing Src homology 2 (SH2) and phosphotyrosine-binding (PTB) domains [53-56]. EGFR-driven Src activation enables ADAM family proteases to cleave to membrane-bound heparin-binding EGF-like growth factor (HB-EGF) from the cell surface and 
drive autocrine EGFR signaling, an important factor in several cancers [57]. The interaction between EGFR and Src is complex. Src binds other receptor tyrosine kinases (RTKs) and activates RTK signaling [58]. Our data show that the phosphorylation of Src expression was increased by stimulation of AR (an EGFR ligand) (Fig. 2E). However, how AR induces the activation of the Src kinase remains unclear. Importantly, the MEK/ERK molecular pathway is downstream of Src signaling transduction; miR-34a targets Src and inhibits the RAF/MEK/ ERK signaling pathway and cell proliferation [29]. Moreover, insulin-like growth factor-1 (IGF-1)-induced VEGF-C upregulation promotes MDA-MB-231 breast cancer cell invasion and lymphatic metastasis via the MAPK/ERK signaling transduction pathway [59]. CCL2 enhances the migration ability of human chondrosarcoma cells in the Ras/Raf/MEK/ERK/ NF- $\kappa B$ signaling pathway [60]. ERK inhibition suppresses the proliferation, migration and invasion of NSCLC cells and inhibits NSCLC tumor growth and metastasis in the ERK signaling pathway [61]. CXCR4/SDF1 promotes matrix metalloproteinase-1 (MMP-1) expression and increases the invasion ability of chondrosarcoma cells through the ERK signaling pathway [62]. Our findings suggest that AR drives chondrosarcoma development in part through the activation of the Src/MEK/ERK signaling pathway, which in turn upregulates VEGF-C expression.

Activation of STAT3 is commonly observed in tumor tissue [63]. Other research demonstrating vascular endothelial cell expression of VEGF-C via the JAK/STAT3 pathway is similar to the findings in our study [64]. We show in the present study that AR stimulation activates Src, which is related to STAT3 phosphorylation in chondrosarcoma cells. In addition, we have shown that MEK and ERK mediate STAT3 activation. STAT3 is therefore a downstream molecule of MEK/ERK. Stimulation with a c-Src inhibitor diminished STAT3 phosphorylation (Fig. 4F), indicating that c-Src signaling occurs upstream of STAT3. Whether STAT3 activation is induced directly from the EGFR in parallel with the Src kinase pathway remains to be clarified.

\section{Conclusion}

In conclusion, we have demonstrated that AR activates Src/MEK/ERL/STAT3 signaling and enhances VEGF-C expression and lymphangiogenesis in chondrosarcoma cells (Fig. 6). AR may therefore be a molecular target for inhibiting lymphangiogenesis and metastasis in chondrosarcoma.

Fig. 6. The pathways involved in ARpromoted VEGF-C expression and lymphangiogenesis. AR enhances VEGF-C production and promotes lymphangiogenesis through the Src/ MEK/ERK/STAT3 signaling pathway. However, how AR induces the activation of the Src kinase is yet to be determined.

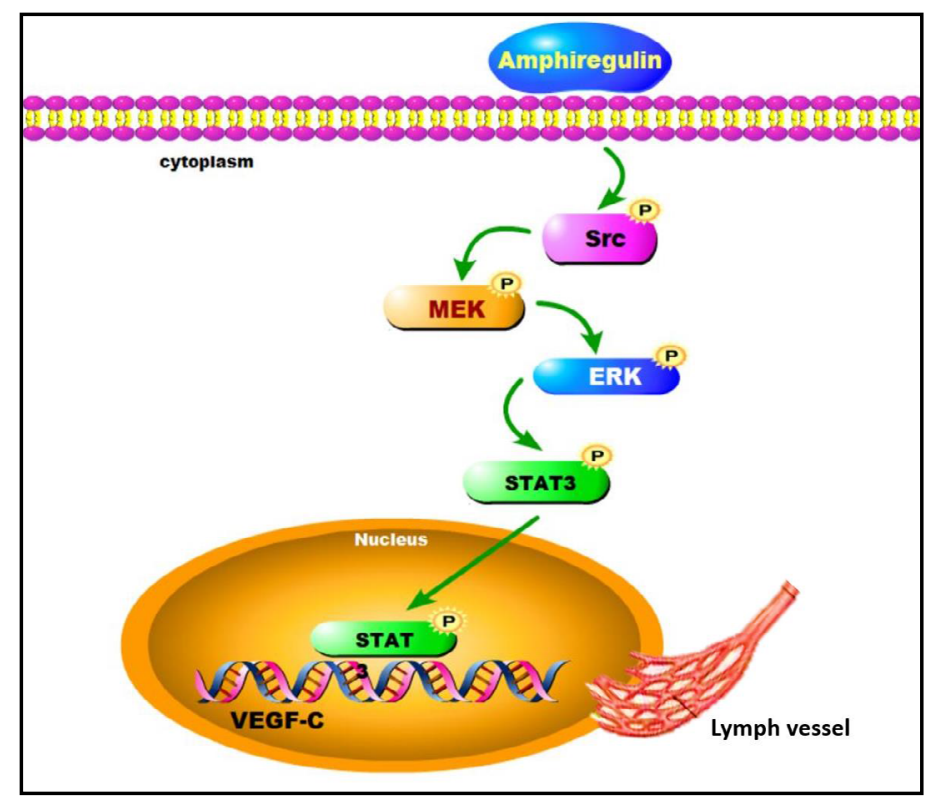




\section{Cellular Physiology Cell Physiol Biochem 2019;52:1-15

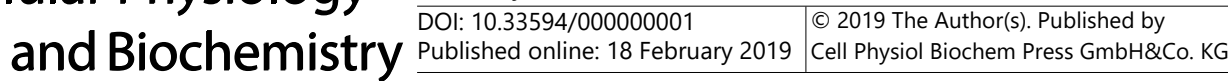 \\ Huang et al.: Amphiregulin Induces VEGF-C-Dependent Lymphangiogenesis}

\section{Acknowledgements}

This work was supported by grants from the Ministry of Science and Technology of Taiwan (MOST 107-2314-B-039-014-; MOST 107-2314-B-039-064-) and China Medical University Hospital (DMR-108-068). We thank Faye L. Shih for helping us to complete this study.

\section{Disclosure Statement}

No conflict of interests exists.

\section{References}

1 Vasudevan HN, Raleigh DR, Johnson J, Garsa AA, Theodosopoulos PV, Aghi MK, Ames C, McDermott MW, Barani IJ, Braunstein SE: Management of Chordoma and Chondrosarcoma with Fractionated Stereotactic Radiotherapy. Front Surg 2017;4:35.

2 De Amorim Bernstein K, DeLaney T: Chordomas and chondrosarcomas-The role of radiation therapy. J Surg Oncol 2016;114:564-569.

3 Terek RM, Schwartz GK, Devaney K, Glantz L, Mak S, Healey JH, Albino AP: Chemotherapy and P-glycoprotein expression in chondrosarcoma. J Orthop Res 1998;16:585-590.

4 Chen JC, Chen YJ, Lin CY, Fong YC, Hsu CJ, Tsai CH, Su JL, Tang CH: Amphiregulin enhances alpha6beta1 integrin expression and cell motility in human chondrosarcoma cells through Ras/Raf/MEK/ERK/AP-1 pathway. Oncotarget 2015;6:11434-11446.

5 Abdul-Aziz MA, Amin AK, El-Rouby DH, Shaker OG: Lymphangiogenesis in Oral Squamous Cell Carcinoma: Correlation with VEGF-C Expression and Lymph Node Metastasis. Int J Dent 2017;2017:7285656.

- 6 Eveno C, Pocard M: VEGF levels and the angiogenic potential of the microenvironment can affect surgical strategy for colorectal liver metastasis. Cell Adh Migr 2012;6:569-573.

7 Oh N, Park JI, Park JH, Kim KS, Lee DR, Park KS: The role of ELK3 to regulate peritumoral lymphangiogenesis and VEGF-C production in triple negative breast cancer cells. Biochem Biophys Res Commun 2017;484:896-902.

8 Spiric Z, Eri Z, Eric M: Lymphatic vessel density and VEGF-C expression as independent predictors of melanoma metastases. J Plast Reconstr Aesthet Surg 2017; DOI:10.1016/j.bjps.2017.06.040.

9 Kuerti S, Oliveira-Ferrer L, Milde-Langosch K, Schmalfeldt B, Legler K, Woelber L, Prieske K, Mahner S, Trillsch F: VEGF-C expression attributes the risk for lymphatic metastases to ovarian cancer patients. Oncotarget 2017;8:43218-43227.

10 Eroglu A, Ersoz C, Karasoy D, Sak S: Vascular endothelial growth factor (VEGF)-C, VEGF-D, VEGFR-3 and D2-40 expressions in primary breast cancer: Association with lymph node metastasis. Adv Clin Exp Med 2017;26:245-249.

- 11 Lin J, Feng J, Jin Y, Yan Z, Lai Z, Peng J: Pien Tze Huang suppresses VEGF-C-mediated lymphangiogenesis in colorectal cancer. Oncol Rep 2016;36:3568-3576.

- 12 Zhu G, Huang Q Huang Y, Zheng W, Hua J, Yang S, Zhuang J, Wang J, Ye J: Lipopolysaccharide increases the release of VEGF-C that enhances cell motility and promotes lymphangiogenesis and lymphatic metastasis through the TLR4- NF-kappaB/JNK pathways in colorectal cancer. Oncotarget 2016;7:73711-73724.

- 13 Wang LH, Lin CY, Liu SC, Liu GT, Chen YL, Chen JJ, Chan CH, Lin TY, Chen CK, Xu GH, Chen SS, Tang CH, Wang SW: CCL5 promotes VEGF-C production and induces lymphangiogenesis by suppressing miR-507 in human chondrosarcoma cells. Oncotarget 2016;7:36896-36908.

14 Yu J, Tao S, Hu P, Wang R, Fang C, Xu Y, Qi D, Wei Z, Zhang J, Tan Q: CCR7 promote lymph node metastasis via regulating VEGF-C/D-R3 pathway in lung adenocarcinoma. J Cancer 2017;8:2060-2068.

- 15 Berasain C, Avila MA: Amphiregulin. Semin Cell Dev Biol 2014;28:31-41.

- 16 Qin L, Tamasi J, Raggatt L, Li X, Feyen JH, Lee DC, Dicicco-Bloom E, Partridge NC: Amphiregulin is a novel growth factor involved in normal bone development and in the cellular response to parathyroid hormone stimulation. J Biol Chem 2005;280:3974-3981. 


\section{Cellular Physiology and Biochemistry}

Cell Physiol Biochem 2019;52:1-15

\begin{tabular}{l|l}
\hline DOI: 10.33594/000000001 & (c) 2019 The Author(s). Published by
\end{tabular}

\begin{tabular}{ll} 
Published online: 18 February 2019 Cell Physiol Biochem Press GmbH\&Co. KG \\
\hline
\end{tabular}

Huang et al.: Amphiregulin Induces VEGF-C-Dependent Lymphangiogenesis

- 17 Zhu J, Siclari VA, Liu F, Spatz JM, Chandra A, Divieti Pajevic P, Qin L: Amphiregulin-EGFR signaling mediates the migration of bone marrow mesenchymal progenitors toward PTH-stimulated osteoblasts and osteocytes. PLoS One 2012;7:e50099.

18 Khelwatty S, Essapen S, Bagwan I, Green M, Seddon A, Modjtahedi H: The impact of co-expression of wildtype EGFR and its ligands determined by immunohistochemistry for response to treatment with cetuximab in patients with metastatic colorectal cancer. Oncotarget 2017;8:7666-7677.

19 Peterson EA, Jenkins EC, Lofgren KA, Chandiramani N, Liu H, Aranda E, Barnett M, Kenny PA: Amphiregulin Is a Critical Downstream Effector of Estrogen Signaling in ERalpha-Positive Breast Cancer. Cancer Res 2015;75:4830-4838.

20 Liu JF, Tsao YT, Hou CH: Amphiregulin enhances intercellular adhesion molecule-1 expression and promotes tumor metastasis in human osteosarcoma. Oncotarget 2015;6:40880-40895.

21 Yamada M, Ichikawa Y, Yamagishi S, Momiyama N, Ota M, Fujii S, Tanaka K, Togo S, Ohki S, Shimada H: Amphiregulin is a promising prognostic marker for liver metastases of colorectal cancer. Clin Cancer Res 2008;14:2351-2356.

22 Tokunaga S, Nagano T, Kobayashi K, Katsurada M, Nakata K, Yamamoto M, Tachihara M, Kamiryo H, Yokozaki H, Nishimura Y: Amphiregulin as a Novel Resistance Factor for Amrubicin in Lung Cancer Cells. Anticancer Res 2017;37:2225-2231.

23 Wang CQ, Huang YW, Wang SW, Huang YL, Tsai CH, Zhao YM, Huang BF, Xu GH, Fong YC, Tang CH: Amphiregulin enhances VEGF-A production in human chondrosarcoma cells and promotes angiogenesis by inhibiting miR-206 via FAK/c-Src/PKCdelta pathway. Cancer Lett 2017;385:261-270.

24 Cheng JC, Chang HM, Xiong S, So WK, Leung PC: Sprouty2 inhibits amphiregulin-induced down-regulation of E-cadherin and cell invasion in human ovarian cancer cells. Oncotarget 2016;7:81645-81660.

25 Tsai HC, Tzeng HE, Huang CY, Huang YL, Tsai CH, Wang SW, Wang PC, Chang AC, Fong YC, Tang CH: WISP-1 positively regulates angiogenesis by controlling VEGF-A expression in human osteosarcoma. Cell Death Dis 2017;8:e2750.

26 Xia X, Lemieux ME, Li W, Carroll JS, Brown M, Liu XS, Kung AL: Integrative analysis of HIF binding and transactivation reveals its role in maintaining histone methylation homeostasis. Proc Natl Acad Sci U S A 2009;106:4260-4265.

27 Stacker SA, Williams SP, Karnezis T, Shayan R, Fox SB, Achen MG: Lymphangiogenesis and lymphatic vessel remodelling in cancer. Nat Rev Cancer 2014;14:159-172.

28 Huang YH, Yang HY, Huang SW, Ou G, Hsu YF, Hsu MJ: Interleukin-6 Induces Vascular Endothelial Growth Factor-C Expression via Src-FAK-STAT3 Signaling in Lymphatic Endothelial Cells. PLoS One 2016;11:e0158839.

29 Yang Y, Ding L, Guo ZK, Zheng XL, Wang LS, Sun HY, Jin ZG, Wang HX: The epigenetically-regulated miR-34a targeting c-SRC suppresses RAF/MEK/ERK signaling pathway in K-562 cells. Leuk Res 2017;55:91-96.

30 Song X, Wei Z, Shaikh ZA: Requirement of ERalpha and basal activities of EGFR and Src kinase in Cdinduced activation of MAPK/ERK pathway in human breast cancer MCF-7 cells. Toxicol Appl Pharmacol 2015;287:26-34.

31 Xiong Y, Huang F, Li X, Chen Z, Feng D, Jiang H, Chen W, Zhang X: CCL21/CCR7 interaction promotes cellular migration and invasion via modulation of the MEK/ERK1/2 signaling pathway and correlates with lymphatic metastatic spread and poor prognosis in urinary bladder cancer. Int J Oncol 2017;51:75-90.

32 Zhao G, Zhu G, Huang Y, Zheng W, Hua J, Yang S, Zhuang J, Ye J: IL-6 mediates the signal pathway of JAK-STAT3-VEGF-C promoting growth, invasion and lymphangiogenesis in gastric cancer. Oncol Rep 2016;35:1787-1795.

33 Fukumoto T, Iwasaki T, Okada T, Hashimoto T, Moon Y, Sakaguchi M, Fukami Y, Nishigori C, Oka M: High expression of Mcl-1L via the MEK-ERK-phospho-STAT3 (Ser727) pathway protects melanocytes and melanoma from UVB-induced apoptosis. Genes Cells 2016;21:185-199.

34 Giuffrida AY, Burgueno JE, Koniaris LG, Gutierrez JC, Duncan R, Scully SP: Chondrosarcoma in the United States (1973 to 2003): an analysis of 2890 cases from the SEER database. J Bone Joint Surg Am 2009;91:1063-1072.

35 Nota SP, Braun Y, Schwab JH, van Dijk CN, Bramer JA: The Identification of Prognostic Factors and Survival Statistics of Conventional Central Chondrosarcoma. Sarcoma 2015;2015:623746.

36 Van Trappen PO, Pepper MS: Lymphatic dissemination of tumour cells and the formation of micrometastases. Lancet Oncol 2002;3:44-52. 


\section{Cellular Physiology Cell Physiol Biochem 2019;52:1-15 \begin{tabular}{ll|l}
\cline { 2 - 3 } and & 이 $10.33594 / 000000001$ & 2019 The Author(s). Published by
\end{tabular} and BiOChemistry Published online: 18 February 2019 Cell Physiol Biochem Press GmbH\&Co. KG \\ Huang et al.: Amphiregulin Induces VEGF-C-Dependent Lymphangiogenesis}

- 37 Tang C, Wang P, Li X, Zhao B, Yang H, Yu H, Li C: Lymph node status have a prognostic impact in breast cancer patients with distant metastasis. PLoS One 2017;12:e0182953.

38 Yang C, Cheng H, Luo G, Lu Y, Guo M, Jin K, Wang Z, Yu X, Liu C: The metastasis status and tumor burdenassociated CA125 level combined with the CD4/CD8 ratio predicts the prognosis of patients with advanced pancreatic cancer: A new scoring system. Eur J Surg Oncol 2017; DOI:10.1016/j.ejso.2017.07.010.

39 Miladi I, Vivier M, Dauplat MM, Chatard M, Besse S, Vidal A, Chassain K, Jean B, Forestier C, Chezal JM, Redini F, Degoul F, Miot-Noirault E: Doxycycline and its quaternary ammonium derivative for adjuvant therapies of chondrosarcoma. Cancer Chemother Pharmacol 2017; DOI:10.1007/s00280-017-3377-7.

40 Tsai CH, Yang DY, Lin CY, Chen TM, Tang CH, Huang YL: Sphingosine-1-phosphate suppresses chondrosarcoma metastasis by upregulation of tissue inhibitor of metalloproteinase 3 through suppressing miR-101 expression. Mol Oncol 2017; DOI:10.1002/1878-0261.12106.

41 Kutzner EA, Park JS, Zaheer S, Inman JC: Tracheal Chondrosarcoma: Systematic Review of Tumor Characteristics, Diagnosis, and Treatment Outcomes with Case Report. Case Rep Oncol Med 2017;2017:4524910.

42 Kim JW, Kim DK, Min A, Lee KH, Nam HJ, Kim JH, Kim JS, Kim TY, Im SA, Park IA: Amphiregulin confers trastuzumab resistance via AKT and ERK activation in HER2-positive breast cancer. J Cancer Res Clin Oncol 2016;142:157-165.

43 Zhao W, Ding G, Wen J, Tang Q Yong H, Zhu H, Zhang S, Qiu Z, Feng Z, Zhu J: Correlation between Trop2 and amphiregulin coexpression and overall survival in gastric cancer. Cancer Med 2017;6:994-1001.

44 Wang B, Yong H, Zhu H, Ni D, Tang S, Zhang S, Wang W, Zhou Y, Zhao W, Ding G, Zhu J, Li X, Feng Z: Abnormal amphiregulin expression correlates with gastric cancer prognosis. Oncotarget 2016;7:76684-76692.

45 Wang L, Wu H, Wang L, Lu J, Duan H, Liu X, Liang Z: Expression of amphiregulin predicts poor outcome in patients with pancreatic ductal adenocarcinoma. Diagn Pathol 2016;11:60.

46 Busser B, Sancey L, Brambilla E, Coll JL, Hurbin A: The multiple roles of amphiregulin in human cancer. Biochim Biophys Acta 2011;1816:119-131.

47 Taverna S, Pucci M, Giallombardo M, Di Bella MA, Santarpia M, Reclusa P, Gil-Bazo I, Rolfo C, Alessandro R: Amphiregulin contained in NSCLC-exosomes induces osteoclast differentiation through the activation of EGFR pathway. Sci Rep 2017;7:3170.

48 Zhang J, Wang Y, Chen X, Zhou Y, Jiang F, Chen J, Wang L, Zhang WF: MiR-34a suppresses amphiregulin and tumor metastatic potential of head and neck squamous cell carcinoma (HNSCC). Oncotarget 2015;6:74547469.

49 Chen Q, Zhou Z, Shan L, Zeng H, Hua Y, Cai Z: The importance of Src signaling in sarcoma. Oncol Lett 2015;10:17-22.

50 Huang YH, Yang HY, Hsu YF, Chiu PT, Ou G, Hsu MJ: Src contributes to IL6-induced vascular endothelial growth factor-C expression in lymphatic endothelial cells. Angiogenesis 2014;17:407-418.

- 51 Ischenko I, Seeliger H, Camaj P, Kleespies A, Guba M, Eichhorn ME, Jauch KW, Bruns CJ: Src tyrosine kinase inhibition suppresses lymphangiogenesis in vitro and in vivo. Curr Cancer Drug Targets 2010;10:546-553.

- 52 Johnson GR, Wong L: Heparan sulfate is essential to amphiregulin-induced mitogenic signaling by the epidermal growth factor receptor. J Biol Chem 1994;269:27149-27154.

- 53 Xu H, Zong H, Ma C, Ming X, Shang M, Li K, He X, Du H, Cao L: Epidermal growth factor receptor in glioblastoma. Oncol Lett 2017;14:512-516.

- 54 Lemmon MA, Schlessinger J: Cell signaling by receptor tyrosine kinases. Cell 2010;141:1117-1134.

- 55 Wee P, Wang Z: Epidermal Growth Factor Receptor Cell Proliferation Signaling Pathways. Cancers (Basel) 2017;9:52.

56 Scaltriti M, Baselga J: The epidermal growth factor receptor pathway: a model for targeted therapy. Clin Cancer Res 2006;12:5268-5272.

57 Hynes NE, Schlange T: Targeting ADAMS and ERBBs in lung cancer. Cancer Cell 2006;10:7-11.

58 Wagner MJ, Stacey MM, Liu BA, Pawson T: Molecular mechanisms of SH2- and PTB-domain-containing proteins in receptor tyrosine kinase signaling. Cold Spring Harb Perspect Biol 2013;5:a008987.

- 59 Zhu C, Qi X, Chen Y, Sun B, Dai Y, Gu Y: PI3K/Akt and MAPK/ERK1/2 signaling pathways are involved in IGF1-induced VEGF-C upregulation in breast cancer. J Cancer Res Clin Oncol 2011;137:1587-1594.

60 Tang CH, Tsai CC: CCL2 increases MMP-9 expression and cell motility in human chondrosarcoma cells via the Ras/Raf/MEK/ERK/NF-kappaB signaling pathway. Biochem Pharmacol 2012;83:335-344. 


\section{Cellular Physiology Cell Physiol Biochem 2019;52:1-15}

\begin{tabular}{ll|ll} 
and Biochemistry & DOl: 10.33594/000000001 & O 2019 The Author(s). Published by \\
Published online: 18 February 2019 & Cell Physiol Biochem Press GmbH\&Co. KG
\end{tabular}

Huang et al.: Amphiregulin Induces VEGF-C-Dependent Lymphangiogenesis

61 Zhang P, Guo X, Li J, Yu S, Wang L, Jiang G, Yang D, Wei Z, Zhang N, Liu J, Sun Y: Immunoglobulin-like transcript 4 promotes tumor progression and metastasis and up-regulates VEGF-C expression via ERK signaling pathway in non-small cell lung cancer. Oncotarget 2015;6:13550-13563.

- 62 Sun X, Wei L, Chen Q, Terek RM: CXCR4/SDF1 mediate hypoxia induced chondrosarcoma cell invasion through ERK signaling and increased MMP1 expression. Mol Cancer 2010;9:17.

63 Buettner R, Mora LB, Jove R: Activated STAT signaling in human tumors provides novel molecular targets for therapeutic intervention. Clin Cancer Res 2002;8:945-954.

64 Miyazaki T, Taketomi Y, Saito Y, Hosono T, Lei XF, Kim-Kaneyama JR, Arata S, Takahashi H, Murakami M, Miyazaki A: Calpastatin counteracts pathological angiogenesis by inhibiting suppressor of cytokine signaling 3 degradation in vascular endothelial cells. Circ Res 2015;116:1170-1181. 